\title{
Pyocele of the lachrymal sac: A late and unusual complication after surgery for a juvenile nasopharyngeal angiofibroma
}

\author{
Philippe Eloy \\ Vincent Bachy \\ Véronique Grulois \\ Bernard Bertrand \\ Department of Otorhinolaryngology, \\ Head and Neck Surgery, University \\ Hospital of Mont-Godinne, Université \\ Catholique de Louvain, Yvoir, Belgium
}

Correspondence: Philippe Eloy University Hospital of Mont-Godinne, Université Catholique de Louvain, 5530, Yvoir, Belgium

Tel +3281423705

Fax +32 81423703

Email philippe.eloy@orlo.ucl.ac.be

\begin{abstract}
JNA (Juvenile nasopharyngeal angiofibroma) is a benign but highly vascular and aggressive tumor that takes its origin in the basisphenoid region close to the sphenopalatine foramen. It occurs invariably in male teenagers. Surgery is the treatment of choice. In the past, external transfacial approaches were recommended. Nowadays endonasal endoscopic approach is performed by experienced teams even for extended tumor. The authors report a case of a pyocele of the lachrymal sac occurring 60 years after a transantral surgery for a JNA. The patient was then successfully operated with an endonasal endoscopic dacryocystorhinostomy using a powered instrumentation and a navigation system. This case confirms the necessity of a long follow-up for all the patients who had a transantral surgery with resection of the medial wall of the maxillary sinus and dissection of the nasolacrymal duct.
\end{abstract}

Keywords: pyocele of the lachrymal sac, complication, surgery for JNA, JNA, endonasal DCR, navigation system

\section{Introduction}

Juvenile nasopharyngeal angiofibroma (JNA) is a rare, benign but highly vascular and aggressive tumor that takes its origin in the basisphenoid region, close to the sphenopalatine foramen. It occurs invariably in male teenagers. Its management consists of a complete surgical resection of the tumor, its insertions and extensions. In the past, external transfacial approaches were recommended (Pryor et al 2005). Nowadays endonasal endoscopic approach is currently performed by experienced teams in endoscopic surgery even for large and extended tumors (Hofmann et al 2005; Marshall and Bradley 2006; Andrade et al 2007; Eloy et al 2007). When the tumor invades the maxillary sinus, the medial wall of the maxillary sinus must be removed. Therefore, the management of the lachrymal pathway could be a challenge. We report the story of a patient operated for a JNA, who experienced a pyocele of the lachrymal sac 60 years later. To our knowledge, this is the first case published in the worldwide literature.

\section{Case report}

A 70-year-old patient came to the outpatient clinic with a right-sided acute dacryocystitis with a trend to fistulization to the skin. In his medical history, he mentioned a transantral surgery for a JNA performed 60 years before. Since then, he was complaining of intermittent and persistent tearing but this was not too impeding. Two months before the consultation in our department, he presented an acute dacryocystitis on the right side, requiring external puncture of the abscess and the prescription of broad spectrum antibiotics for 15 days. One month after this episode, the disease recurred with a strong trend to a fistulization to the skin (Figure 1a). That time, he was referred to the ENT department for 
management. Nasal endoscopy displayed a highly distorted anatomy, sequel of the previous surgery. There was no evidence of recurrence of the JNA but the mucosa in the right posterior ethmoid sinus was hyperplasic. It was also confirmed that the nasolacrymal duct was cut in the past. No opening of the nasolacrymal duct could be identified. There was no pus in the nasal cavity (Figure 1b).

A sinus computed tomography (CT) scan demonstrated the extension of the previous surgery with a resection of the posterior nasal septum and the medial wall of the maxillary sinus. The right ethmoid and sphenoid sinuses were opened. There was no recurrence of the JNA. There was no paranasal mucocoele. The right lachrymal sac was dilated (Figures 1c and 1d).

Because of the recurrence of the ophthalmologic disease despite a recent antibiotic treatment at adequate dosage and duration and because of a trend to fistulization, we decided to perform a dacryocystorhinostomy in emergency. We chose the endonasal approach assisted with a navigation system (BrainLAB, VectorVision ${ }^{\circledR}$, France) to identify the right place of the lachrymal sac (Figure 2). The surgery was performed under general anaesthesia using the powered instrumentation $(-2.9 \mathrm{~mm}$ diamond drill; Medtronic Inc., Brussels, Belgium). Pus was aspirated during the surgery. Culture did not yield any bacteria despite numerous neutrophils in the secretions. Because of the size of the opening (wide opening) and the absence of a concomittant canalicular obstruction, we did not put a silicone intubation.

The postoperative period was uneventful. 3 weeks later, the patient was free of symptom (Figure 3a). A fluorescein
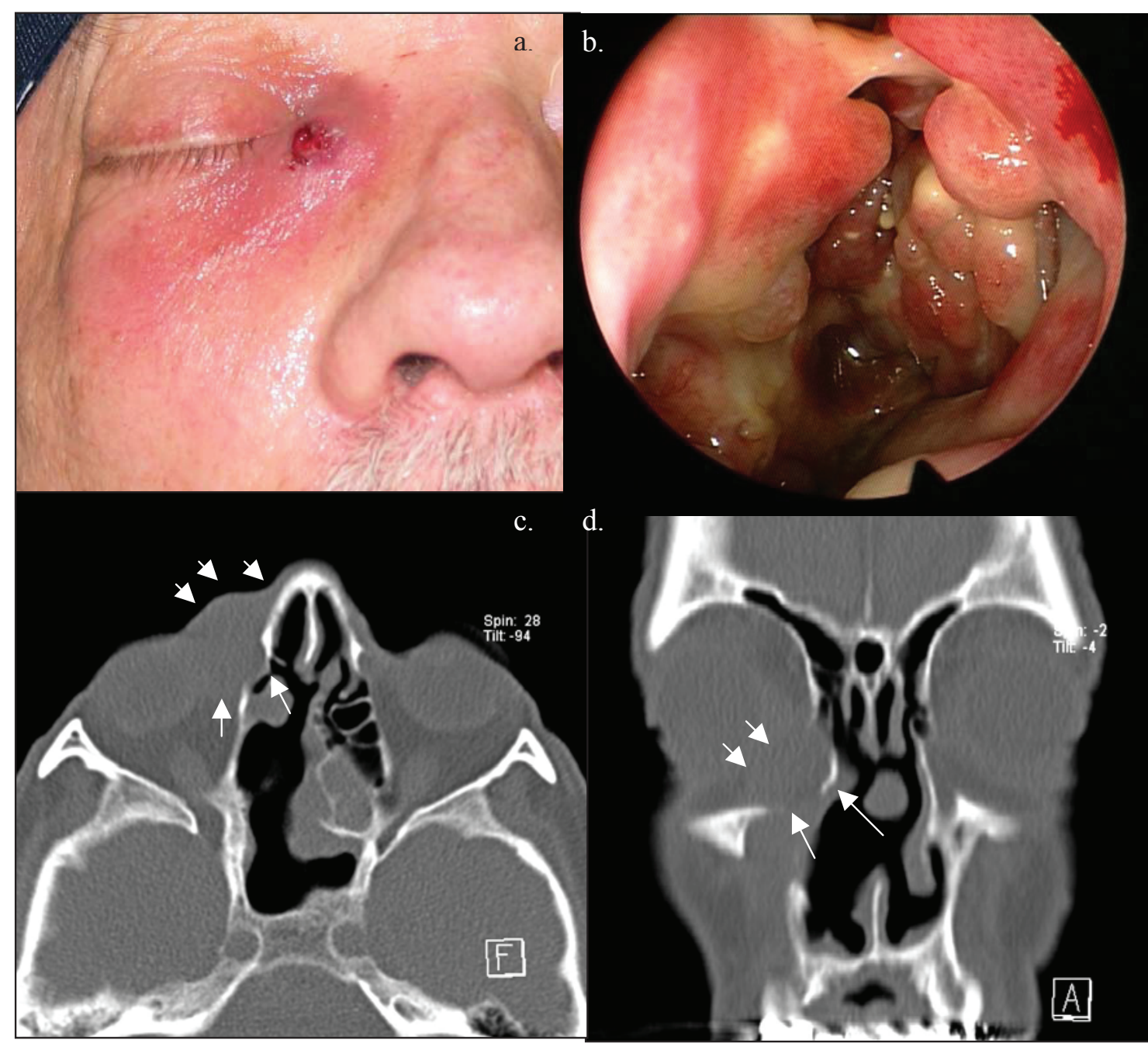

d.
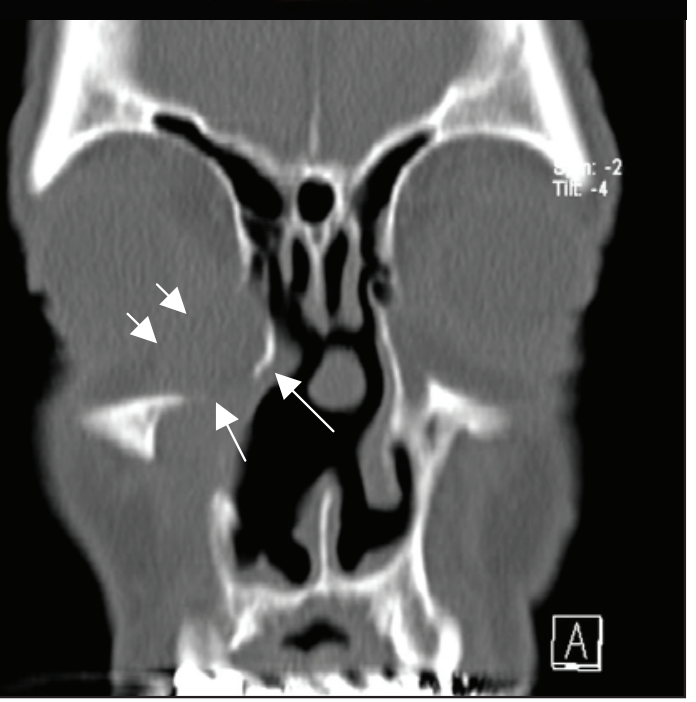

Figure I a: Macroscopic preoperative view of the patient - fistulization of a right acute dacryocystitis. b: endoscopy of the right nasal cavity - distorted anatomy. c: preoperative axial sinus CT scan - the lachrymal sac is indicated by the white arrows. d: preoperative coronal sinus CT scan - the sac is showed by the arrows. 


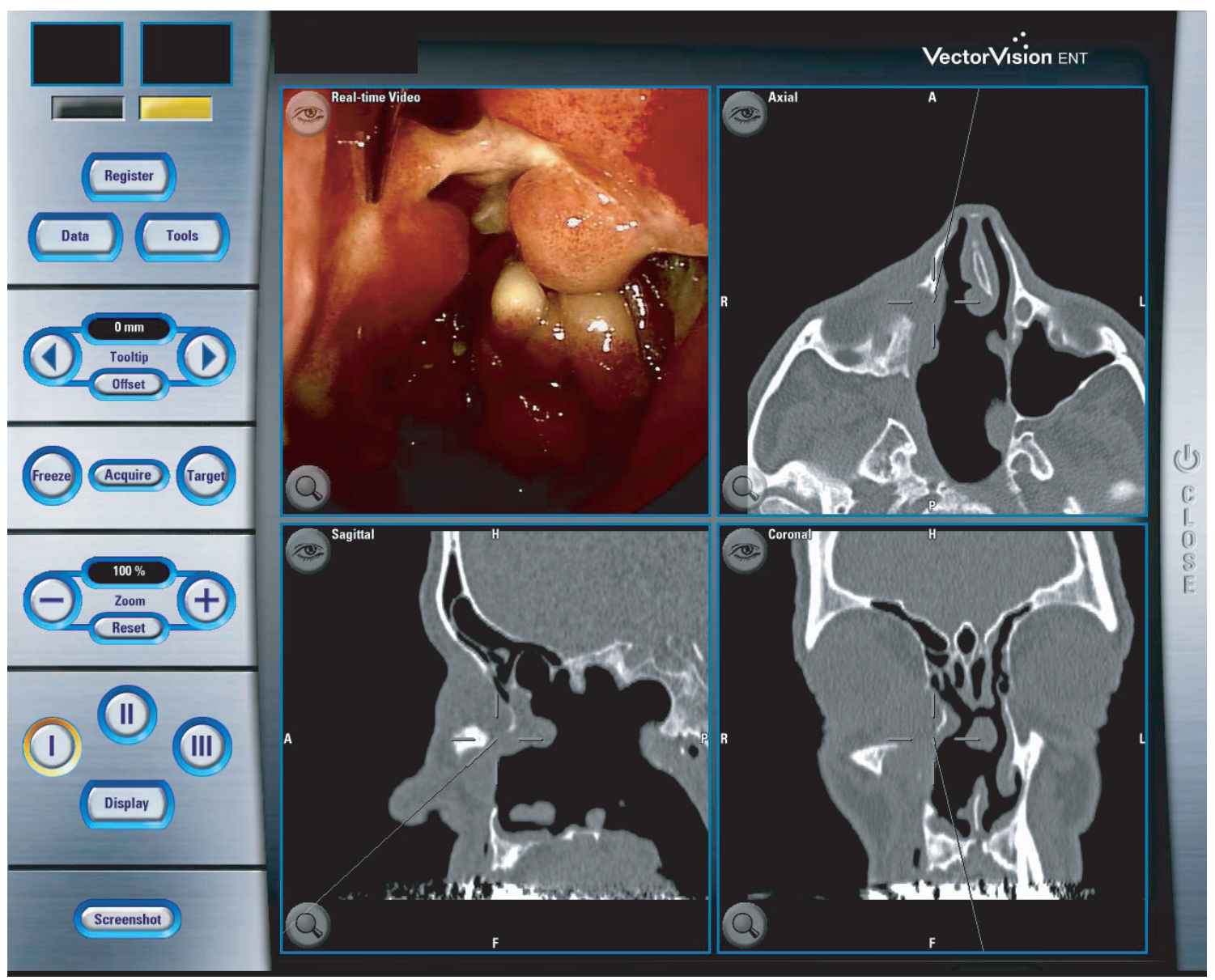

Figure 2 Screen of the navigation system. Right image on the top: real time video. Left image on the top (sinus CT scan: axial cut). Right image at the bottom (sinus CT scan: sagittal cut). Right image at the bottom (Sinus CT scan: coronal cut).

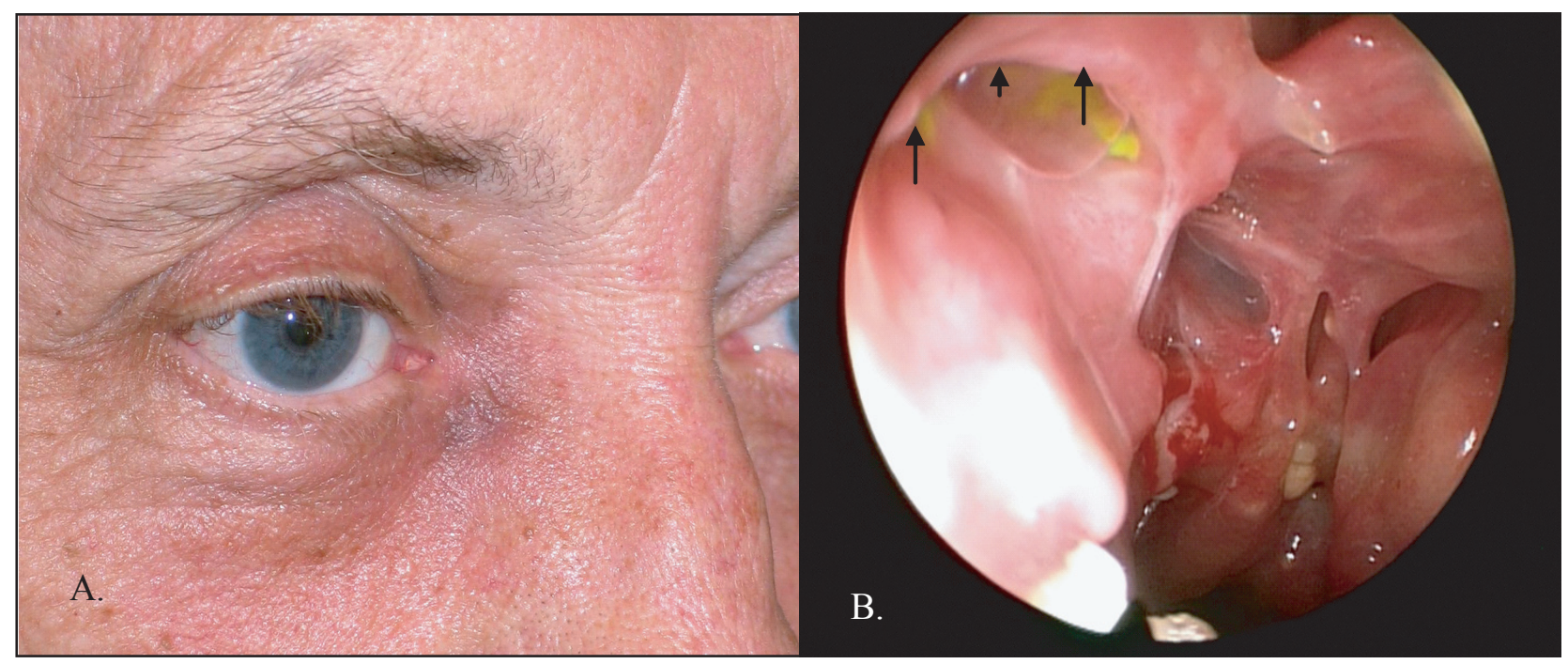

Figure 3 Postoperative findings: A: Macroscopic view of the patient 3 weeks after the surgery. B: Nasal endoscopic, large surgical opening; positive fluorescein dye disappearance test (black arrows). 
dye disappearance test confirmed the patency of the surgical stoma (Figure 3b).

Six months later, the patient is still free of symptom. At the nasal endoscopy, the stoma is wide, with no crust or granulation tissue in the nasal cavity.

\section{Discussion}

When the surgery for JNA involves the maxillary sinus, the resection of the medial wall of the maxillary sinus is indicated. Management of the nasolacrymal duct may be a challenge. Classically simple section of the duct is sufficient but in some cases, the section is done very close to the sac. Consequently, the patient can experience intermittent and persistent isolated epiphora. This problem did not draw the attention until infection occurred. In the present case, it did 60 years after the surgery.

A mucocoele of the lachrymal sac results from a combination of a complete occlusion of the nasolacrymal duct and the presence of mucoid secretions within the sac. In case of an acute bacterial infection, this can lead to a fistulization to the skin.

At that stage, the treatment usually consists of wide broad spectrum antibiotics in conjunction with an external drainage of the "abscess". When the symptoms recur, dacryocystorhinostomy is indicated. It can be performed via an external or an endonasal approach.

External dacryocystorhinostomy (DCR) is a well known and standardized surgical procedure, usually performed by the ophthalmologists (Dolman 2003). Acute infection is a relative contraindication to do it because the skin incision could heal poorly.

Endonasal DCR is a viable alternative to the external approach (Eloy et al 1995). Infection is not a contraindication to perform an endonasal DCR but an expertise in endonasal endoscopic surgery is mandatory and a sinus CT scan must be asked preoperatively to assess the anatomy, particularly when the patient reports a story of facial trauma or previous sinus surgery for tumour. In our case, the anatomy was highly distorted, sequel of the previous surgery for JNA. To skip this problem, a navigation system was used (BrainLAB, VectorVision ${ }^{\circledR}$ ). Indeed it provided peroperative information about the exact location of the instruments and the lachrymal sac. If such an expensive and sophisticated equipment is not available, a light source can be inserted into the canalicular system to locate by transillumination the lachrymal sac. We also used the powered instrumentation as it was described by Wormwald (2002). That allowed us to drill out the frontal process of the maxilla in order to expose widely the lachrymal sac. At the end of the procedure, a wide opening was achieved. Syringing of the lachrymal pathway by both inferior and superior canaliculi confirmed the absence of a blockage at the level of the common canaliculus. Therefore, no silicone intubation was used.

Three weeks after the surgery, the patient was free of symptoms. The swelling of the medial canthus had disappeared. There was no persistent fistula to the skin. 9 months later, the patient was still free of symptoms.

A question that remains unanswered is whether or not the pyocele is a late complication of the surgery for JNA.

An argument against is the delay of 60 years between the surgery and the onset of the acute infection. On the other hand, arguments for are the following: Blockage of the lachrymal pathway is a disease that occurs more commonly in women. Our case occurred in a male. Local factors could have contributed to the development of the pathology. The patient himself is convinced that there is a link between the surgery and the lachrymal disease. He experienced for many years an intermittent and persistent epiphora that did not draw his attention until infection occurred. Mucocoele of the lachrymal sac needs time for its development. External surgery for JNA with a complete removal of the sinus mucosa, leads to the formation of crusts and fibrosis in the operated cavities. This can contribute to a stenosis of the remnant part of the "lachrymal duct".

\section{Conclusion}

This anecdotal case report demonstrates that surgery of the maxillary sinus comprising the resection of the medial wall of the maxillary sinus can be complicated, even many years later, by ophthalmologic problems such as an obstruction of the nasolacrymal duct and a pyocele of the lachrymal sac.

The ENT specialist and ophthalmologist should be aware of such a complication. Endonasal DCR is the easiest way to manage an acute dacryocystitis resistant to the conventional medical treatment. When the endonasal anatomy is highly distorted, the use of a navigation system is recommended. If the surgeon does not have such equipment and/or enough expertise in endoscopic endonasal surgery, the endonasal approach should be regarded as too hazardous and the external approach must be considered.

\section{Disclosure of interest}

We disclose any financial or commercial support that could lead to a conflict of interest. 


\section{References}

Andrade NA, Pinto JA, de Oliveira Nobrega M, et al. 2007. Exclusively endoscopic surgery for juvenile nasopharyngeal angiofibroma. Otolaryngol Head Neck Surg, 137:492-6.

Dolman PJ. 2003. Comparison of external dacryocystorhinostomy with non laser endonasal dacryocystorhinostomy. Ophthalmology, 110:78-84.

Eloy P, Bertrand B, Martinez M, et al. 1995. Endonasal dacryocystorhinostomy: indications, technique and results. Rhinology, 33:229-33.

Eloy P, Watelet JB, Hatert AS, et al. 2007. Exclusively endoscopic surgery for juvenile nasopharyngeal angiofibroma. Otolaryngol Head Neck Surg, 137:492-6.

Hofmann T, Bernal-Sprekelsen M, Koele W, et al. 2005. Endoscopic resection of juvenile angiofibromas - long term results. Rhinology, 43:282-9.

Marshall AH, Bradley PJ. 2006. Management dilemmas in the treatment and follow-up of advanced juvenile nasopharyngeal angiofibroma. $O R L$ J Otorhinolaryngol Relat Spec, 68:273-8.

Pryor SG, Moore EJ, Kasperbauer JL. 2005. Endoscopic versus traditional approaches for excision of juvenile nasopharyngeal angiofibroma. Laryngoscope, 115:1201-7.

Wormwald PJ. 2002. Powered endoscopic dacryocystorhinostomy. Laryngoscope, 112:69-72. 
